\title{
AMPK agonist AICAR improves cognition and motor coordination in young and aged mice
}

\author{
Tali Kobilo, ${ }^{1,3}$ Davide Guerrieri, ${ }^{1,3}$ Yongqing Zhang, ${ }^{2}$ Sarah C. Collica, ${ }^{1}$ Kevin G. Becker, ${ }^{2}$ \\ and Henriette van Praag ${ }^{1,4}$
}

\begin{abstract}
${ }^{1}$ Neuroplasticity and Behavior Unit, Laboratory of Neurosciences, National Institute on Aging, National Institutes of Health, Baltimore, Maryland 21224, USA; ${ }^{2}$ Research Resources Branch, Intramural Research Program, National Institute on Aging, National Institutes of Health, Baltimore, Maryland 21224, USA
\end{abstract}

\begin{abstract}
Normal aging can result in a decline of memory and muscle function. Exercise may prevent or delay these changes. However, aging-associated frailty can preclude physical activity. In young sedentary animals, pharmacological activation of AMP-activated protein kinase (AMPK), a transcriptional regulator important for muscle physiology, enhanced spatial memory function, and endurance. In the present study we investigated effects of AMPK agonist 5-aminoimidazole-4carboxamide riboside (AICAR) on memory and motor function in young (5- to 7-wk-old) and aged (23-mo-old) female $\mathrm{C} 57 \mathrm{Bl} / 6$ mice, and in young (4- to 6-wk-old) transgenic mice with muscle-specific mutated AMPK $\alpha 2$-subunit (AMPKDN). Mice were injected with AICAR (500 mg/kg) for 3-14 d. Two weeks thereafter animals were tested in the Morris water maze, rotarod, and open field. Improved water maze performance and motor function were observed, albeit at longer duration of administration, in aged (14-d AICAR) than in young (3-d AICAR) mice. In the AMPK-DN mice, the compound did not enhance behavior, providing support for a muscle-mediated mechanism. In addition, microarray analysis of muscle and hippocampal tissue derived from aged mice treated with AICAR revealed changes in gene expression in both tissues, which correlated with behavioral effects in a dose-dependent manner. Pronounced up-regulation of mitochondrial genes in muscle was observed. In the hippocampus, genes relevant to neuronal development and plasticity were enriched. Altogether, endurance-related factors may mediate both muscle and brain health in aging, and could play a role in new therapeutic interventions.
\end{abstract}

Normal aging is generally associated with incremental physical and mental frailty. Memory function declines gradually, together with structural and functional changes in the rodent and human hippocampus, a brain area important for learning and memory (Granger et al. 1996; Hillman et al. 2008). In particular, synaptic plasticity, vascularization, neurogenesis, and neurotrophin levels are reduced in rodents (Black et al. 1990; Mattson and Magnus 2006) and performance on hippocampus-dependent tasks declines (Erickson and Barnes 2003). Concomitantly, a progressive loss of skeletal muscle mass and strength (sarcopenia) is observed (Baumgartner et al. 1992; Peterson et al. 2012). Muscle changes may be attributed to loss of motor neuron units, changed contractility, and reduced mitochondrial function (Lang et al. 2010). Although both physical and cognitive decline can likely not be prevented in the elderly, lifestyle interventions such as exercise may attenuate these processes both in the brain (Hillman et al. 2008; van Praag 2008) and periphery (Mattson 2012; Pedersen and Febbraio 2012). However, aging-associated frailty or disease may preclude physical activity. Use of compounds that may mimic, at least in part, the effects of exercise (Narkar et al. 2008; Kobilo et al. 2011) could be a suitable alternative.

Energy balance is modulated by AMPK, a transcriptional regulator. More specifically, AMPK is a "master regulator" of metabolism. Upon activation, it phosphorylates many metabolic enzymes, and so decreases anabolic pathways that consume ATP,

\footnotetext{
${ }^{3}$ These authors contributed equally to this paper.

${ }^{4}$ Corresponding author

E-mail vanpraagh@mail.nih.gov

Article is online at http://www.learnmem.org/cgi/doi/10.1101/lm.033332.113.
}

such as protein biosynthesis, while activating catabolic pathways that generate ATP, such as glucose uptake. It also concurrently phosphorylates transcription factors and coactivators that regulate gene expression, for example those relevant to mitochondrial function and biogenesis (Hardie 2011a,b; Hardie et al. 2012). Recently, AMPK has been identified as an important factor regulating muscle fiber contractile gene expression and endurance (Narkar et al. 2008). Treatment with AMPK agonist AICAR increased running stamina by $45 \%$ in sedentary animals (Narkar et al. 2008) and enhanced spatial memory function in young mice (Kobilo et al. 2011). As AICAR has very low permeability across the blood-brain barrier (Marangos et al. 1990), effects on stamina and cognition are likely indirect. Indeed, muscle may be considered a secretory organ that, upon stimulation with endurance factors or exercise, could release so-called myokines, such as BDNF, LIF, IL-6, which may exert influence throughout the body, including the brain (Pedersen et al. 2003; Sakuma and Yamaguchi 2011; Mattson 2012; Pedersen and Febbraio 2012).

An increase in the cellular AMP/ATP ratio, signaling that the cell's energy status needs to be replenished, activates AMPK (Hardie 2011a). With aging, ATP production under low-energy conditions is reduced in muscle (Chen Scarabelli et al. 2008), possibly due to declining mitochondrial function and content (Mansouri et al. 2006; Corsetti et al. 2008; Figueiredo et al.

This article is distributed exclusively by Cold Spring Harbor Laboratory Press for the first 12 months after the full-issue publication date (see http://learnmem. cshlp.org/site/misc/terms.xhtml). After 12 months, it is available under a Creative Commons License (Attribution-NonCommercial 3.0 Unported), as described at http://creativecommons.org/licenses/by-nc/3.0/. 
2008). In addition, old rats reportedly have decreased AMPK activity in muscle upon acute stimulation with AICAR and treadmill exercise (Reznick et al. 2007), and exhibit reduced basal AMPK phosphorylation in muscle (Qiang et al. 2007; however, see Thomson and Winder 2009). Therefore, in the present study, we aimed to determine whether aging-related decline in memory and motor function could be attenuated by muscle-mediated activation of AMPK. To identify possible underlying mechanisms, microarray analysis of hippocampal and muscle tissue was performed. In addition, mice with selective inactivation of AMPK $\alpha 2$ (AMPK-DN mice), eliminating essentially all AMPK activity in skeletal muscle (Zong et al. 2002; Zwetsloot et al. 2008), were evaluated following treatment with AICAR. Altogether, our results show that AICAR treatment benefits motor and memory function in aged mice, possibly by increasing expression of muscle mitochondrial and hippocampal neural plasticity genes.

\section{Results}

\section{Effects of AICAR on spatial memory and motor behavior}

\section{Young mice}

AMPK agonist AICAR or saline was injected for $3 \mathrm{~d}$ in female (5- to 7 -wk old) wild type (wt) C57Bl/ 6 mice ( $n=10$ per group). Body weight was measured weekly and did not differ between the groups $\left(F_{(1,18)}=0.51, P>0.49\right)$. Two weeks later mice were trained in the water maze with four trials per day over $7 \mathrm{~d}$. One-way ANOVA with repeated measures (Days) showed that water maze learning was modestly enhanced in mice that received AICAR $(500 \mathrm{mg} / \mathrm{kg})$ for $3 \mathrm{~d}$ (ACR3), 2 wk prior to the onset of behavioral testing (saline, $n=10$; ACR3, $n=10$ ) (Fig. 1A). Specifically, a marginal effect for latency $\left(F_{(1,18)}=3.22, P=0.08\right)$ and a significant effect for dis-
A
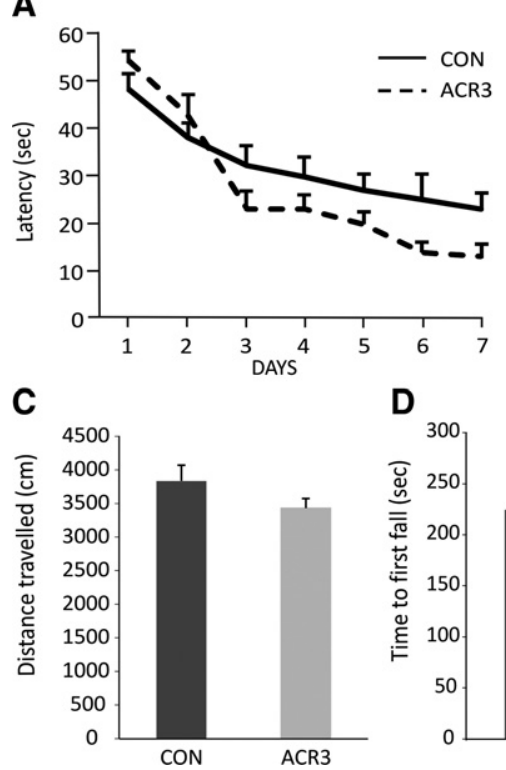

B

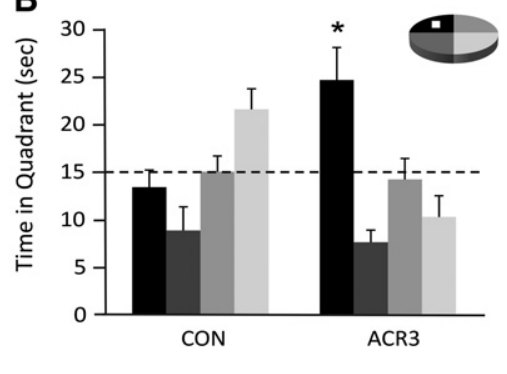

E

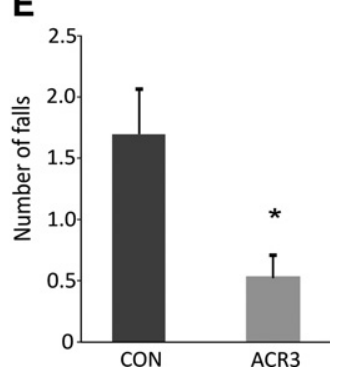

Figure 1. Water maze performance and motor behavior in mice treated with saline (CON) or AICAR (ACR3, $500 \mathrm{mg} / \mathrm{kg}$ ) for $3 \mathrm{~d}$. Mice were trained for $7 \mathrm{~d}$ with four trials per day in the Morris water maze, 2 wk after injections. $(A)$ ACR3 mice had a trend of shorter latency to the platform over training days than CON mice $(P=0.08)$. (B) Only the ACR3 mice showed a significant preference for the target zone as compared to all other quadrants of the pool in the 4-h probe trial $\left({ }^{*} P<0.006\right)$. ( $\left.C-E\right)$ To assess motor behavior mice were tested in the open field and rotarod. (C) Distance traveled in the open field showed a modest trend toward reduction for ACR injected mice. $(D)$ Latency to the first fall in the rotarod did not differ between the groups. (E) In the rotarod the ACR3 group had significantly less total falls $\left({ }^{*} P<0.015\right)$. Error bars indicate SEM. tance $\left(F_{(1,18)}=4.55, P<0.05\right)$ to the platform were observed. There was no difference between the groups in swim speed $\left.F_{(1,18)}=0.20, P>0.90\right)$. Furthermore, compound-treated mice preferred the platform quadrant as compared to all other quadrants in probe trials at $4 \mathrm{~h}$ after the last training trial on day 7, as evidenced by one-way ANOVA over quadrants $\left(F_{(3,36)}=12.29\right.$, $P<0.0001)$, followed by specific comparisons between the time spent in the target quadrant and time in each of the other quadrants $(P<0.006)$, whereas saline-treated mice did not exhibit such a preference (Fig. 1B). These findings are consistent with previous studies where treatment with AICAR for $7 \mathrm{~d}$ enhanced retenof spatial memory (Kobilo et al. 2011)

Motor activity and coordination were tested in the open open field did not differ between groups $\left(F_{(1,18)}=2.02, P>\right.$ the first fall over three trials was rotarod the average latency to 0.28 ) (Fig. 1D). However, the total number of falls within the 5 -min testing period per trial was significantly less in the ACR3 mice than in the control group $\left(F_{(1,18)}=7.19, P<0.02\right)$, suggesting improved motor coordination (Fig. 1E).

\section{Aged mice}

Muscle AMPK levels decline with aging (Reznick et al. 2007) raisficient in aged mice. Indeed, upon administration of saline or (ACAR $(500 \mathrm{mg} / \mathrm{kg}$ ) for 3 (ACR3), 7 (ACR7), or 14 (ACR14) d, ory. Specifically, mice were trained in the water maze over $12 \mathrm{~d}$ groups in latency to the platform $\left(F_{(3,28)}=0.56, P>0.64\right)$ or swim speed $\left(F_{(3,28)}=0.68, \quad P>0.57\right)$ analyzed by one-way ANOVA with repeated measures over days (Fig. 2A). However, the 4-h probe trial after the last training trial on day 12 revealed a significant preference for the platform quadrant as compared to the other three quadrants of the pool only in ACR14 mice, as evidenced by one-way ANOVA over quadrants $\left(F_{(3,24)}=3.61\right.$, $P<0.027)$, followed by specific comparisons between the time spent in the target quadrant and the dwell time in each of the other quadrants $(P<0.03)$ (Fig. 2B). Interestingly, prolonged administration of AICAR ( $14 \mathrm{~d})$ in young mice is not beneficial (Kobilo et al. 2011), suggesting that a shift in the dose-response/duration of administration occurs with aging.

Motor behavior was also evaluated in the aged mice. Specifically, locomotor activity was examined in the open field arena. A significant effect of treatment on activity was observed $\left(F_{(3,28)}=4.51\right.$, $P<0.01)$. Specific comparisons indicated that the ACR7 $(5330 \pm 437 \mathrm{~cm})$ and ACR14 $(6080 \pm 534 \mathrm{~cm})$ groups traveled a greater distance in the open field during the 30-min testing period than CON $(4216 \pm 231 \mathrm{~cm})$ mice $(P<0.05)$. The ACR14 group also differed significantly from ACR3 (5004 $\pm 244 \mathrm{~cm}$ ) (Fig. 2C). To determine whether the compound may have an anxiolytic effect the distance traveled in the center of the arena 
A

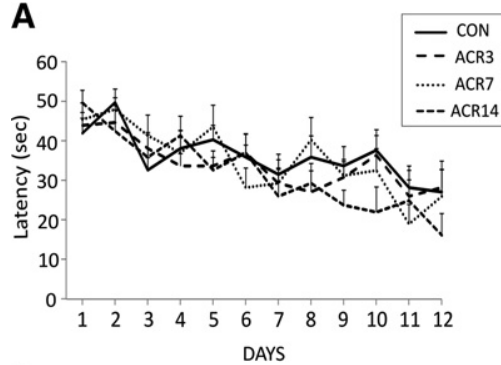

B

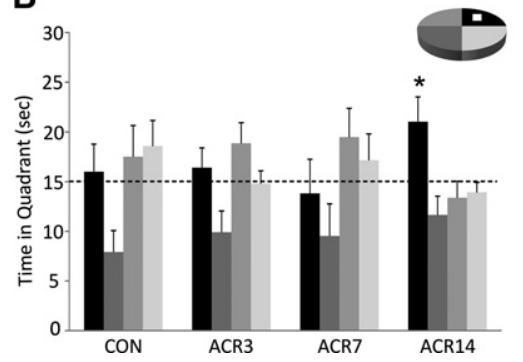

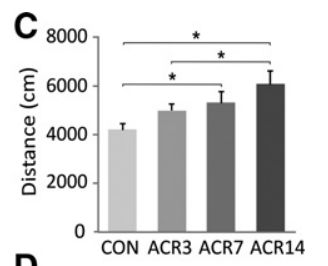

D

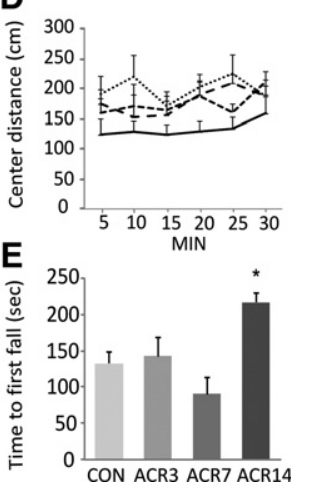

Figure 2. Water maze and motor behavior in aged $C 57 \mathrm{BI} / 6$ mice (23-mo old) treated with AICAR for 3 (ACR3), 7 (ACR7) or 14 (ACR14) d or injected with saline $(C O N)$. $(A)$ Mice were tested in the Morris water maze with four trials per day for $12 \mathrm{~d}$. There was no difference between the groups in acquisition of the task $(P>0.65)$. (B) In the probe trial conducted $4 \mathrm{~h}$ after the last training trial, ACR14 mice showed a clear preference for the target quadrant as compared to all other quadrants $\left({ }^{*} P<0.03\right)$. (C) Locomotor activity was examined in the open field arena over $30 \mathrm{~min}$. ACR7 and ACR14 mice traveled a greater distance in the open field than CON mice, and ACR14 also differed significantly from ACR3 ( $P<0.05)$. $(D)$ Distance traveled in the center of the open field did not differ between the groups. $(E)$ Motor coordination and strength were tested in the rotarod. Mice injected with AICAR for $14 \mathrm{~d}$ showed a longer latency to the first fall than the other groups $\left({ }^{*} P<0.02\right)$. Error bars indicate SEM.

was also analyzed; however, no significant difference between the groups was observed $\left(F_{(3,28)}=2.10, P>0.12\right)$ (Fig. 2D). Improved rotarod performance was observed in aged mice treated with AICAR. Aged mice injected with AICAR for $14 \mathrm{~d}$ showed a longer latency to the first fall in the rotarod than all other groups $\left(F_{(3,27)}=3.81, P<0.02\right)$ (Fig. $2 \mathrm{E}$ ). Together these results indicate improved coordination and activity in aged mice treated with AICAR.

\section{Behavior in AMPK-DN mice}

To evaluate whether muscle AMPK may play a role in the enhanced performance we tested young mice overexpressing an inactive AMPK $\alpha 2$ catalytic subunit driven by the muscle specific MCK promoter (AMPK-DN, verified by PCR, Fig. 3A). Overexpression of the inactive AMPK $\alpha 2$ subunit eliminates essentially all AMPK activity in skeletal muscle (Zwetsloot et al. 2008). Female wild type (wt) and transgenic (tg) littermates (4- to 6-wk old) were injected with saline $(n=12 \mathrm{wt}, n=10 \mathrm{tg})$ or $\operatorname{AICAR}(n=10 \mathrm{wt}, n=$ $7 \mathrm{tg}$ ) for $3 \mathrm{~d}$ and trained $2 \mathrm{wk}$ later in the water maze with four trials per day over $5 \mathrm{~d}$ (Fig. 3B). Two-way analysis of variance with repeated measures (Genotype $\times$ Treatment $\times$ Days) showed that there was no interaction between the groups in acquisition of the task $\left(F_{(1,35)}=0.11, P>0.74\right)$ and no main effects of genotype or treatment $(P>0.05)$. Upon evaluation of retention of platform location in the probe trial $4 \mathrm{~h}$ after the last training trial, drug-treated wt mice (ACR-) preferred the target quadrant, as evidenced by a significant one-way ANOVA over quadrants $\left(F_{(3,36)}=4.19, P<0.01\right)$, followed by specific post-hoc comparisons between the time spent in the target quadrant and time in

each of the other quadrants $(P<0.03)$. In addition, a trend toward significance was observed for time spent in the target quadrant in the ACR- versus the ACR+ group $\left(t_{(15)}=2.01, P=0.06\right)$, supporting the notion that the compound was effective in enhancing memory retention in wt but not in tg mice (Fig. 3C).

Mice treated with saline $(n=8 \mathrm{wt}, n=6 \mathrm{tg})$ or AICAR $(n=7$ $\mathrm{wt}, n=7 \mathrm{tg}$ ) for $3 \mathrm{~d}$ were also tested in the rotarod and open field. Distance traveled in the open field over $30 \mathrm{~min}$ did not differ between wt and $\mathrm{tg}$ saline $(4495 \pm 251 \mathrm{~cm}$, wt; $5146 \pm 588 \mathrm{~cm}, \mathrm{tg})$ and AICAR $(4622 \pm 480 \mathrm{~cm}$, wt; $4894 \pm 352 \mathrm{~cm}$, tg) treated mice $\left(F_{(1,24)}=0.57, P>0.64\right)$. In addition, neither the average latency to the first fall over three trials in saline ( $246 \pm 22 \mathrm{sec}$, wt; $251 \pm 22$ sec, tg) and AICAR $(243 \pm 21 \mathrm{sec}, \mathrm{wt} ; 235 \pm 20 \mathrm{sec}, \operatorname{tg})\left(F_{(1,24)}=\right.$ $0.09, P>0.76)$ treated mice, nor the total number of falls $\left(F_{(1,24)}=0.03, P>0.87\right)$, differed between the groups.

\section{Microarray analysis}

Microarray analysis of hippocampal and gastrocnemius muscle tissue derived from aged mice treated with AICAR for $3 \mathrm{~d}$ (ACR3), $7 \mathrm{~d}$ (ACR7), or $14 \mathrm{~d}$ (ACR14) or saline (CON) was consistent with the behavioral findings. The most pronounced changes in gene expression were found in the ACR7 and ACR14 groups in both tissues. Principal component analysis (DIANE6, http:// www.grc.nia.nih.gov/branches/rrb/dna/diane_software.pdf) showed a parallel dose-response pattern in both gastrocnemius muscle and hippocampus. Further analysis suggested bidirectional effects between the muscle and the brain. Muscle sample gene analysis revealed effects on brain-related genes, such as up-regulation of neuronal fate commitment. In addition, muscle-related genes, important for formation of striated muscle thin filaments, contraction, cholesterol homeostasis, and fatty acid biosynthesis, exhibited elevated expression. Furthermore, components of mitochondrion and of mitochondrial inner membrane, mitochondrial activity of NADH dehydrogenase, complex IV, and complex I were up-regulated in muscle samples. In the hippocampus up-regulation of components of the mitochondrion and endoplasmic reticulum, as well as genes involved in metabolic processes, glycolysis, oxidoreductase activity, and lipid biosynthesis were observed. However, the majority of up-regulated genes in the hippocampus were related to neuronal development (Fig. 4A-D).

In both tissues for ACR7 and ACR14 samples, the majority of the top 30 up-regulated genes are the same, albeit different for muscle and hippocampus. Specific analysis of the top 10 up-regulated genes in the muscle (Fig. 4C) revealed that eight genes were identically up-regulated in the ACR7 and ACR14 groups, in comparison to control. Increased gene expression relevant to energy metabolism in muscles was observed, for example, for NDUFA5, NDUFB9, NDUFB4 (codes for subunits of complex I of the respiratory chain, which transfers electrons from NADH to ubiquinone [Walker 1992], 3.27-, 3.07-, and 2.82-fold, respectively), SDHD (Complex II, 2.53-fold), DLD, ATP5A1 (encodes a subunit of mitochondrial ATP synthase, that catalyzes ATP synthesis [Pedersen and Amzel 1993], 2.65- and 3.11-fold, respectively), and USMG5 (integral to the mitochondrial membrane, 3.01-fold increase).

Analysis of the top down-regulated genes in muscle (Fig. 4C) revealed genes involved in fatty acid metabolism such as LPIN2 (Donkor et al. 2007), -1.53-fold, as well as genes whose inhibition increases insulin sensitivity, such as RCAN2 (Sun et al. 2011), -1.32-fold. Indeed, inactivation of the RCAN2 gene in mice also ameliorates age- and diet-induced obesity by causing a reduction in food intake (Sun et al. 2011). Down-regulation was also found for ERRFI1 (reduces cell proliferation and muscle growth by inhibiting EGFR protein [Segatto et al. 2011], 

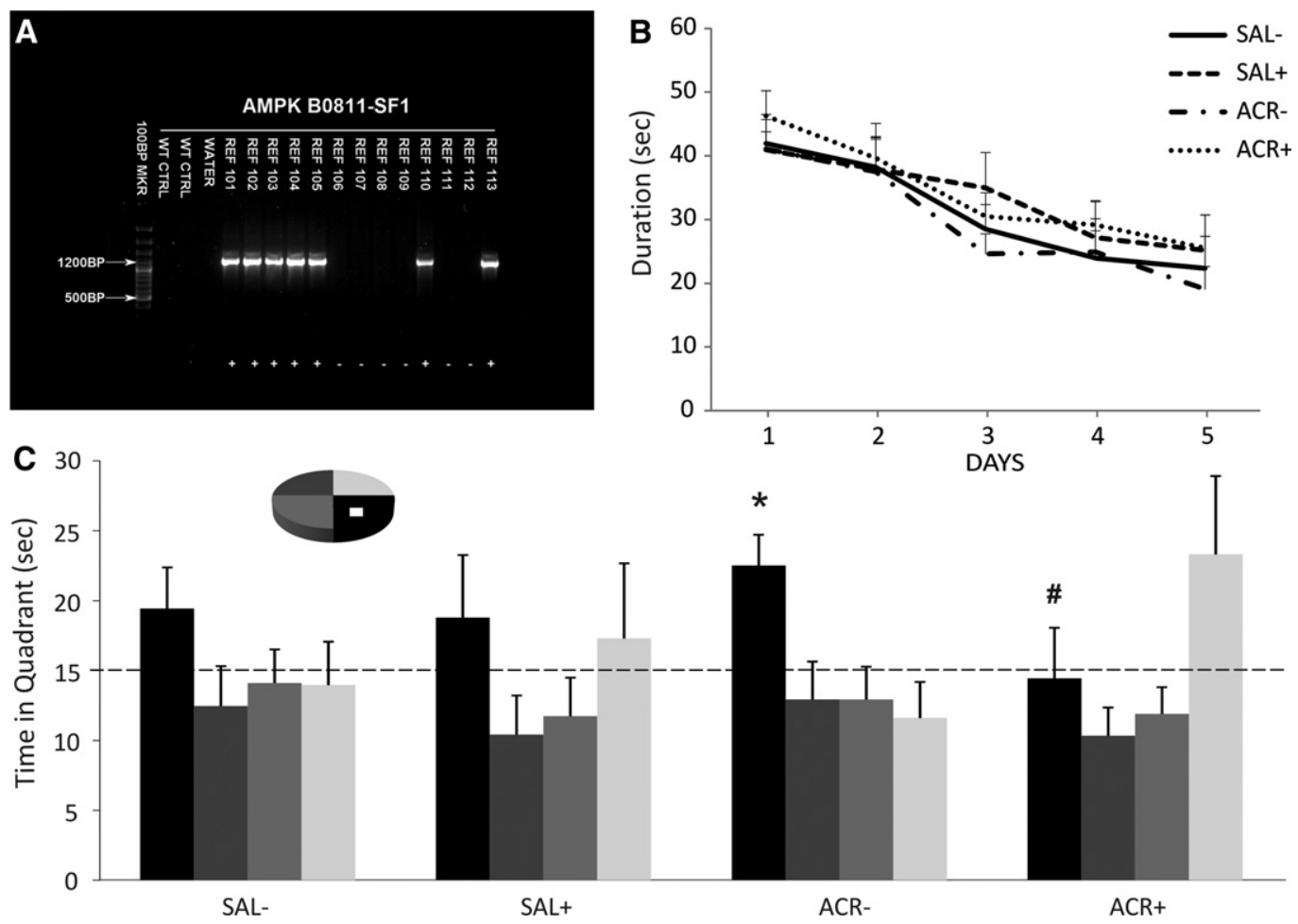

Figure 3. Effect of AICAR on cognition in AMPK-DN mice. (A) PCR analysis of isolated tail DNA was performed to identify AMPK-DN (tg) or wt mice. (B) Water maze performance in AMPK-DN mice (+) or wild type mice (-) treated with Saline (SAL) or AICAR (ACR, $500 \mathrm{mg} / \mathrm{kg}$ ) for $3 \mathrm{~d}$. Mice were trained for $5 \mathrm{~d}$ with four trials per day in the Morris water maze, $2 \mathrm{wk}$ after injections. There was no difference between the groups in acquisition of the task. (C) In the probe trial $4 \mathrm{~h}$ after the last training trial drug-treated wt mice that were injected with ACR (ACR-) showed a significant preference for the platform quadrant compared to the other quadrants $\left({ }^{*} P<0.03\right)$. The mice treated with saline (wt, $S A L-$, and $\left.\operatorname{tg}, S A L+\right)$ and the tg mice treated with AICAR (ACR+) showed no retention of platform location. In addition, there was a trend toward a significant difference in time spent in the target quadrant between ACR - and ACR + mice $\left({ }^{\#} P=0.06\right)$, further supporting memory retention enhancement by AICAR in wt mice. Error bars indicate SEM.

-1.66-fold), SPARC (authophagy and senescence [Nakamura et al. 2012], -1.21-fold), and MYH7 (encodes for MHC $\beta$, specific for slow fibers [van Rooij et al. 2009], -1.27 -fold).

In the hippocampus, even if diffusely up-regulated as a gene class, mitochondria-related genes were a minority in the top 30 enriched genes (ALDOA [2.15-fold], MDH2 [1.79-fold], COX4 [1.32-fold]), whereas distinct elevations were present for neuronal development related genes (Fig. 4D): ATXN10 (neuronal development, 2.15-fold [März et al. 2004]), YWHAG (synaptic plasticity, neuronal differentiation, 2.05-fold [Cabeza-Arvelaiz et al. 2011]), MAPK3 (proliferation and differentiation, 1.25-fold), RTN4 or NOGO, a ligand for NGR1 (axonal regulation/growth inhibition, neuroprotection [Teng and Tang 2008, 2013], and dendritic spine stabilization [Mironova and Giger 2013], 2.07-fold), GOLGA2 (axonogenesis, 1.94-fold), and FGF13 (encodes for a protein member of the fibroblast growth factor [FGF] family, 2.03fold). FGFs possess broad mitogenic and cell survival activities, and are involved in a variety of biological processes, including embryonic development cell growth (Wu et al. 2012). Moreover, we found enrichment of genes relevant to trophic factors, such as PTGDS and PTPRE (2.78- and 2.42-fold, respectively). The protein encoded by $P T G D S$ is a glutathione-independent prostaglandin $\mathrm{D}$ synthase that catalyzes the conversion of prostaglandin $\mathrm{H} 2$ to prostaglandin D2 (Nagata et al. 1991), which functions as a neuromodulator as well as a trophic factor in the central nervous system (Nagata et al. 1991). In addition, PTPRE encodes for a member of the protein tyrosine phosphatase (PTP) family. PTPs are known to be signaling molecules that regulate a variety of cellular processes, including cell growth and differentiation (Avraham et al. 2000).
Furthermore, among the top down-regulated genes in the hippocampus, the microarray analysis revealed genes whose inhibition results in an increase in neurogenesis and neuronal development: GRIT (regulates dendritic spine morphology and strength [Nakamura et al. 2002], -1.48-fold), MEF2C (skeletal muscle myogenesis and neurogenesis, by negatively regulating transcription factor activity [McDermott et al. 1993; Ornatsky and McDermott 1996], -1.25-fold), TENM4 (neurite outgrowth, interacts with the DNA-binding transcriptional repressors, and regulates transcription factor activity, -1.32 -fold). Moreover, down-regulation of Pde1a, a calmodulin-dependent phosphodiesterase with a role in learning and memory, was observed ( -1.27 -fold). Inhibition of Pdela is a target for treatment of cognitive disorders (Fig. 4D; Xu et al. 2011).

\section{Microarray validation}

Microarray analysis of CON and ACR14 samples ( $n=4$ per group) of both hippocampus and gastrocnemius was used to select four genes presenting a Selector value equals 2 ( $z$-ratio $\geq 1.5$, $P$-value $\leq 0.05, \mathrm{fdr} \leq 0.3$, and average signal intensity $>0$ ). Realtime PCR was used to validate microarray results and was conducted for ATP5A1, SDHD (showed a Selector equals 2 in the gastrocnemius group) and for COX4, and MAPK3 (showed a Selector equals 2 in the hippocampus group). The qPCR analysis revealed consistent trends toward increased mRNA expression in ACR14 samples. In particular, a close to significant increase in ATP5A1 (1.89-fold, $\left.t_{(6)}=2.25, P=0.066\right)$ and in SDHD (1.79-fold, $t_{(6)}=$ $2.35, P=0.057)$ in gastrocnemius samples was observed, while in 
A

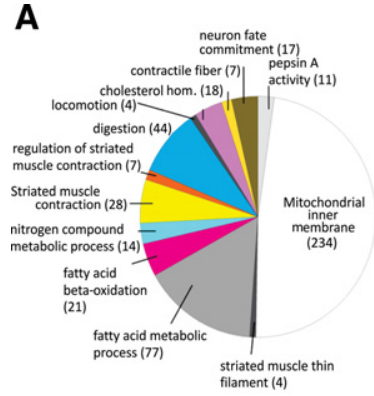

B

\begin{tabular}{|c|c|c|c|c|}
\hline \multirow[b]{2}{*}{ Symbol } & \multicolumn{2}{|c|}{ ACR7 vs CON } & \multicolumn{2}{|c|}{ ACR14 vs CON } \\
\hline & fold change & Zratio & fold change & Zratio \\
\hline Atp5a1 & 3.11 & 10.09 & 2.99 & 9.57 \\
\hline Ndufb9 & 3.07 & 10.05 & 3.22 & 10.09 \\
\hline Ndufa5 & 3.27 & 9.71 & 3.34 & 9.81 \\
\hline Usmg5 & 3.01 & 8.12 & 3.31 & 9.14 \\
\hline Sdhd & 2.53 & 7.60 & 2.65 & 7.95 \\
\hline Dld & 2.65 & 7.44 & 2.89 & 8.36 \\
\hline Ndufb4 & 2.52 & 7.07 & 2.82 & 8.08 \\
\hline Errfi1 & -1.66 & -4.06 & -1.45 & -2.96 \\
\hline Sparc & -1.21 & -1.98 & -1.40 & -3.09 \\
\hline Rcan2 & -1.18 & -2.37 & -1.33 & -3.11 \\
\hline Lpin1 & -1.32 & -1.89 & -1.53 & -3.23 \\
\hline Myh7 & -1.27 & -2.55 & -1.52 & -3.71 \\
\hline
\end{tabular}

D

\begin{tabular}{ccccc} 
& \multicolumn{2}{c}{ ACR7 vs CON } & \multicolumn{2}{c}{ ACR14 vs CON } \\
\hline Symbol & fold change & Zratio & fold change & Zratio \\
\hline Atxn10 & 2.15 & 6.92 & 2.00 & 6.09 \\
Rtn4 & 2.07 & 6.55 & 1.87 & 5.48 \\
Golga2 & 1.94 & 6.03 & 1.93 & 5.81 \\
Ywhag & 2.05 & 5.98 & 1.90 & 5.61 \\
Aldoa & 1.98 & 5.51 & 2.01 & 6.14 \\
Cox411 & 1.32 & 1.34 & 1.32 & 2.24 \\
Mapk3 & 1.25 & 1.86 & 1.30 & 1.84 \\
\hline \hline Pde1a & -1.27 & -2.57 & -1.46 & -3.66 \\
Kcnh1 & -1.46 & -3.58 & -1.54 & -4.03 \\
Tenm4 & -1.32 & -2.91 & -1.57 & -4.25 \\
Mef2c & -1.25 & -2.23 & -1.62 & -4.64 \\
Grit & -1.48 & -3.76 & -1.69 & -4.98 \\
\hline
\end{tabular}

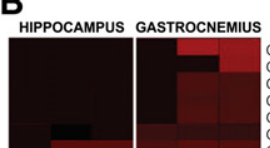

S00006099 TRICARBOXYLIC ACID CYCLE ON0000510 ATP DEPENDENT PROTEOLYSIS 00006635 FATTY ACID BETA OXIDATION G00006118 ELECTRON TRANSPORT 00061122 MITOCHONDRIAL ELECTRON TRANSPOR S00006096 GLYCOLYSIS
G00005783 ENDOPLASMATIC RETICULUM G00005743 MITOCHONDRIAL INNER MEMBRANE
INOC G00005739 MITOCHONDRION G00008137 NADH DEHYDROGENASE UBIQUINONE ACTIVITY G00016491 OXIDOREDUCTASE ACTIVITY G00004129 CYTOCHROME C ACTIVITY 00016651 OXIDOREDUCTASE ACTIVITY ACTING ON NADH S00004984 OLFACTORY RECEPTOR ACTIVITY
G00004872 RECEPTOR ACTIVITY 000004871 SIGNAL TRANSDUCER ACTIVITY G00004930 G PROTEIN COUPLED RECEPTOR ACTIVITY

E

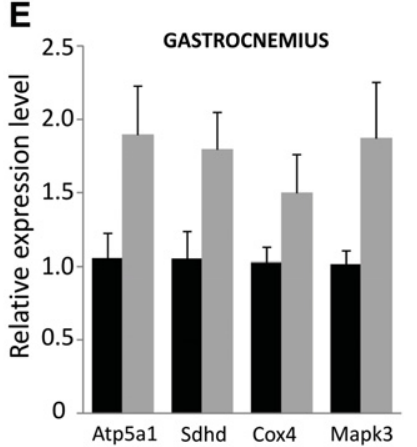

$F$

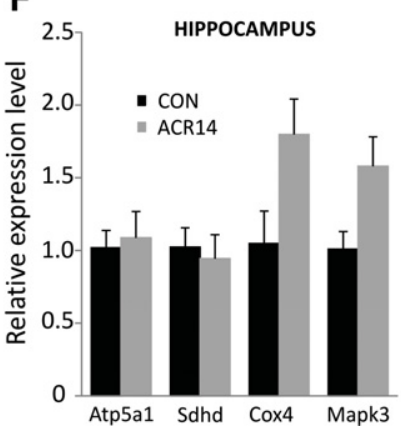

Figure 4. Microarray analysis of hippocampus and muscle tissue derived from aged mice treated with saline (CON) or AICAR for $3 d$ (ACR3), $7 d$ (ACR7), or $14 d$ (ACR14). (A) Gene set analysis of gastrocnemius tissue. Increased expression of mitochondrial inner membrane and metabolism related genes was observed. Each section represents the number of up-regulated genes of the respective function. $(B)$ The heat-map illustrates the expression of the selected signature genes derived from microarray analysis. Up-regulated genes are colored in red and down-regulated genes are colored in green. (C) Top up-regulated and down-regulated genes by ACR7 and ACR14 in gastrocnemius. The tables list the most highly up-regulated genes related to energy metabolism and neuronal development; up-regulation is expressed as fold change compared to control and as z-ratio for each gene. $(D)$ Top up-regulated and down-regulated genes by ACR7 and ACR14 in hippocampus. The tables list the most highly up-regulated genes related to energy metabolism and neuronal development; up-regulation is expressed as fold change compared to control and as z-ratio for each gene. $(E)$ Relative gene expression level and validation. GPCR analysis of expression levels of four genes in ACR14 samples compared to CON, in gastrocnemius. $(F)$ Relative gene expression level and validation. qPCR analysis of expression levels of four genes in ACR14 samples compared to CON, in hippocampus. $N=4$ per group. Error bars indicate SEM.

hippocampal samples an increase was found for COX4 (1.80-fold, $\left.t_{(6)}=2.29, P=0.062\right)$ and MAPK3 (1.58-fold, $t_{(6)}=2.44, P=$ 0.051) (Fig. 4E,F).

\section{Discussion}

The results demonstrate that the AMPK agonist AICAR increased spatial memory and improved motor function in young and old mice. The effects of AICAR were dependent on the duration of administration. In young mice, brief treatment $(3 \mathrm{~d})$ was effective whereas aged animals required more compound injections (14 d). We propose that the beneficial effects of AICAR may be mediat- ed by muscle-related mechanisms. This is supported by the finding that, in female transgenic mice with a muscle-specific mutated AMPK $\alpha 2$-subunit, the compound did not enhance behavior. Microarray analysis was consistent with the behavioral results, revealing parallel duration effects on the expression of genes in the hippocampus and muscle. Altogether, this study suggests that AMPK can be activated pharmacologically to improve motor and cognitive function.

Age, duration, and route of administration appear to play an important role in the effects of AICAR on behavior. Previous studies have shown that direct application of this compound to the brain does not have beneficial effects. Intracerebral infusion of AICAR impaired memory function (Dash et al. 2006), and in vitro application reduced longterm potentiation in hippocampal slices (Potter et al. 2010). AICAR has very low ability to cross the blood-brain barrier (Marangos et al. 1990), suggesting that observed effects of peripheral administration are likely indirect. Systemic administration of AICAR for $10 \mathrm{~d}$ improved maze learning in calorie-restricted mice (Dagon et al. 2006). We recently found that in young wild type mice $7 \mathrm{~d}$ of AICAR treatment improved retention of spatial memory in the water whereas longer treatment for $14 \mathrm{~d}$ was not beneficial and may even be considered detrimental (Kobilo et al. 2011; Kobilo and van Praag 2012). In these studies a 2 -wk interim between AICAR administration and behavioral tests was implemented to determine if changes may last beyond acute administration. In the present experiments brief administration (3 d) also enhanced performance in young mice in the water maze when training commenced 2 wk later. Interestingly, in aged mice, $14 \mathrm{~d}$ rather than $3 \mathrm{~d}$ of treatment were needed to observe cognitive benefits.

The fact that more AICAR is needed to elicit effects in aged mice is supported by other studies. Activation of AMPK induced by muscle contraction is repressed in old muscle (Ljubicic and Hood 2009). In addition, AICAR and exercise increase AMPK $\alpha 2$ activity in the muscle of young but not old rats (Reznick et al. 2007), suggesting a deficiency in AMPK sensitivity in aged tissues. However, in young mice with muscle specific knockdown of the AMPK $\alpha 2$ receptor, memory function did not differ from wild type saline treated controls. Future experiments evaluating additional learning paradigms in this mouse model may be needed. In addition, other studies showed that wild type and AMPK-DN mice do not differ in glucose uptake and fatty acid oxidation in skeletal muscle under standard (sedentary) conditions (Miura et al. 2009), suggesting that AMPK activation may not be essential for physiological functions at basal level. AICAR administration did not benefit neural plasticity in the absence of muscle- 
mediated signaling pathways. Consistently, a mouse model of muscular dystrophy showed that overexpressing nitric oxide, which is regulated by AMPK (Fryer et al. 2000), in muscle benefits structural brain plasticity (Deng et al. 2009). Altogether, muscle-related factors may influence cognitive function.

AICAR administration resulted in enhancement in motor coordination, mainly in the aged, postmenopausal (Nelson et al. 1982), female mice, as reflected in the open field and rotarod tests. Similar to the effects on cognition, the aged mice required more days of treatment. Indeed, in the aged mice longer durations of administration ( 7 and $14 \mathrm{~d}$ ) increased activity in the open field, albeit most pronounced with the longest duration. In addition, $14 \mathrm{~d}$ of administration reduced the latency to the first fall from the rotarod in aged animals. These findings are consistent with Narkar et al. (2008) who reported that treatment with AICAR for 4 wk enhances running endurance, as well as with a recent report in a mouse model for Duchenne muscular dystrophy, where the compound was associated with improved rotarod performance and grip strength which was further enhanced by exercise (Bueno Júnior et al. 2012).

Both muscle and hippocampus showed changes in gene expression in aged mice as a result of treatment with AICAR. In the hippocampus, there was increased expression of similar metabolic processes as in muscle. Glycolysis-related genes were also up-regulated in both muscle and brain. In parallel with muscle gene analysis, increased hippocampal expression of neuronal maturation and anti-apoptosis-related genes was observed, as well as a down-regulation of genes exerting a negative regulation on neurogenesis and neuronal development. In muscle, a notable effect was on mitochondrial gene expression. Mitochondria are key regulators of longevity (Bratic and Trifunovic 2010) and become less numerous with age, and membrane potential decreases. Mice with higher proton conductance across skeletal muscle inner mitochondrial membrane live longer (Bratic and Trifunovic 2010). In muscles, the increase in energy metabolism is evidenced by the up-regulation of genes directly involved in mitochondrial electron transport chain: complex I (NDUFA5, NDUFB9, NDUFB4) and complex IV, and also an up-regulation of complex II (SDHD); moreover, also Krebs Cycle is enhanced, both by complex II itself and by the AcCoA indirectly produced by the overexpression of DLD (Harris et al. 1997). AICAR enhanced expression of mitochondria related genes in the hippocampus, consistent with a role of energy metabolism related factors such as PGC1 $\alpha$ in synaptic plasticity (Cheng et al. 2012); however, the main increase in gene expression in the hippocampus was for neural plasticity related genes such as ATXN10, RTN4, and YWHAG.

Thus, in both young and aged mice, it can be considered that stimulation of muscle AMPK activity by AICAR benefits cognition. Although exercise can activate AMPK, in cases of aging and neurodegenerative disease physical activity may not be possible. Administration of AICAR may "rejuvenate" both motor and memory function in aged mice. Understanding the relationship between muscle and brain may lead to new interventions to maintain cognitive function throughout the lifespan.

\section{Materials and Methods}

\section{Subjects and drug treatment}

Young (5- to 7-wk old, $n=20$ ) and aged (23-mo old, $n=32$ ) female C57BL/6J mice (Jackson Laboratory, Bar Harbor, ME) were housed under standard conditions, three mice per cage, with food and water ad libitum. Young mice $(n=10$ per group) were injected intraperitoneally (i.p.) with 5-aminoimidazole-4carboxamide-1- $\beta$-D-ribofuranoside (AICAR, Toronto Research Chemicals Inc.) dissolved in saline, $500 \mathrm{mg} / \mathrm{kg} / \mathrm{day}$, or saline vehicle, for $3 \mathrm{~d}$ (ACR3). Aged mice ( $n=7-9$ per group) were injected with saline (CON) or AICAR for 3 (ACR3), 7 (ACR7), or 14 (ACR14) d. All mice received a total of 14 daily injections. Mice were tested $2 \mathrm{wk}$ later in the water maze, open field, and rotarod. Young mice were perfused $26 \mathrm{~d}$ later and the aged mice $33 \mathrm{~d}$ after the last saline or drug injection.

Female transgenic mice overexpressing an inactive AMPK $\alpha 2$ catalytic subunit (termed AMPK dominant negative [AMPK-DN]) on a C57BL/6 background were used (from Dr. Morris J. Birnbaum, MD, University of Pennsylvania). Wild type (wt) and transgenic (tg) litter mates (4- to 6-wk old) were treated with saline ( $n=12 \mathrm{wt}, n=10 \mathrm{tg})$ or AICAR $(500 \mathrm{mg} / \mathrm{kg})(n=10$ wt, $n=7 \mathrm{tg}$ ) for $3 \mathrm{~d}$ and tested in the Morris water maze, open field, and rotarod $2 \mathrm{wk}$ later. Mice were perfused $24 \mathrm{~d}$ after the last drug injection. AMPK-DN mice were identified by PCR analysis of isolated tail DNA by use of primers specific for the mutated $A M P K \alpha 2$ gene (Fig. 3A).

\section{Behavioral testing}

Mice were trained in the Morris water maze ( $5 \mathrm{ft}$ diameter) to find a platform hidden $5 \mathrm{~mm}$ below the surface of water made opaque with white nontoxic paint (Morris et al. 1982). It should be noted that a 5 -ft-diameter pool is likely more challenging than the 4-ft-diameter pool, typically used with mice (Terry 2009), and may be preferable for memory enhancement experiments. Starting points were changed for each of the four daily trials. A trial lasted either until the mouse had found the platform or for a maximum of $60 \mathrm{sec}$. Mice rested on the platform for $10 \mathrm{sec}$ after each trial. Upon completion of training, when the average latency to the platform for at least one of the groups was 20-30 sec, a probe trial was performed. Specifically, the platform was removed for a 60 -sec probe trial $4 \mathrm{~h}$ after the last training trial. Platform latency, swim path, and speed were recorded (Anymaze, Stoelting Co.).

In the open field test, mice were placed in the center of the open field arena and allowed to freely move while being tracked by an automated tracking system (Activity Monitor Version 4, MED Associates). Aged mice were also tested twice, $4 \mathrm{~d}$ apart, on a rotarod (Med-Associates), at $20 \mathrm{rpm}$ for $300 \mathrm{sec}$. For the aged mice the average latency to fall for the two tests was calculated. The total number of falls within 5 min was not measured in the old mice due to their advanced age and possible frailty. Young mice were tested once, in accelerating paradigm of $2.5-25 \mathrm{rpm}$ in $300 \mathrm{sec}$. The latency to the first fall in each 5 -min period was recorded three consecutive times.

\section{Tissue collection}

Animals were deeply anesthetized with isofluorane and perfused transcardially with $0.9 \% \mathrm{NaCl}$ solution. Hippocampus and gastrocnemius muscle were quickly removed, and frozen on dry ice. Tissue was stored at $-80^{\circ} \mathrm{C}$ for microarray analysis.

\section{Microarray analysis}

Microarray analysis was performed on hippocampal and muscle tissue derived from four aged mice per group (CON, ACR3, ACR7, ACR14). RNA isolation was carried out using the Qiagen RNeasy Mini Kit for animal tissues (Qiagen, Inc.) following the manufacturer's instructions and hybridized to MouseRef- 8 v1 Expression beadchips (Illumina) following protocols listed on the Gene Expression and Genomics Unit of the NIA (http://www. grc.nia.nih.gov/branches/rrb/dna/index/protocols.htm). Microarray fluorescent signals were extracted using an Illumina BeadArray 500GX reader. The signals on each sample are normalized by $\log z$-transformation to obtained $z$-scores and tests for distributions as previously described (Cheadle et al. 2003). Correlation analysis, sample clustering analysis, and principal component analysis include all of the probes and were performed to identify/exclude any possible outliners. The resulting dataset was next analyzed with JMP6, a spreadsheet-based microarray analysis program. After normalization by $\log z$-transformation, 
the statistically significant gene list for each comparison condition was selected upon the conditions of (1) the probe signal detection $P$-value $\leq 0.02$, (2) one way ANOVA over sample groups was $P<0.05$, (3) pairwise $Z$-test $P<0.05$, (4) false discovery rates are $<0.30$, and (5) gene expression changes measured by $z$-ratio were not less than 1.5 the mean of their absolute values. Further clustering/correlation analyses were done based on these selection criteria. Gene set enrichment analysis used gene expression change values (z-ratio) for the average of all of the genes on the microarray. Parametric analysis of gene set enrichment (PAGE) was used (De et al. 2010) for gene set analysis. Gene Sets include the MSIG database (http://www.broadinstitute.org/ gsea/msigdb/collection_details.jsp\#C2, Gene Ontology Database [http://www.geneontology.org/]); GAD human disease and mouse phenotype gene sets (De et al. 2010; Zhang et al. 2010) were used to explore functional level changes. The data discussed in this article have been deposited in NCBI's Gene Expression Omnibus (Edgar et al. 2002) and are accessible through GEO Series accession number GSE50873 (http://www.ncbi.nlm.nih. gov/geo/query/acc.cgi?acc=GSE50873).

\section{Real-time PCR}

Real-time PCR to validate the Illumina microarray data was performed on the mRNA samples previously described $(n=4$ per group). Reverse transcription was carried out on $1 \mu \mathrm{g}$ of RNA using qScript cDNA Supermix (Quanta Bioscience) according to manufacturer protocol; primers for the selected genes ATP5A1, SDHD, COX4, MAPK3, and HSP9O were purchased from Integrated DNA Technologies using IDT online primer designer. The specific sequence of each of the primers was as follows:

ATP5A1: rev. 5'-GGGCTCCAGTTTGTCAAGATA-3', fwd. 5'CGTCTGACCGAGTTGCTAAA-3';

SDHD: rev. 5'-TCACGAATGGTCGAACCTAAC-3', fwd. 5'-GG TGGGCAGAATGTCTTCTAA-3';

MAPK3: rev. 5'-CTCTTAGGGTTCTTTGACAGTAGG-3', fwd. 5'-CTACACCAACCTCTCGTACATC-3';

COX4: rev. 5'-GATCGAAAGTATGAGGGATGGG-3', fwd. 5'-TG AATGGAAGACAGTTGTGGG-3'; and

endogenous control, HSP90: rev. 5'-CCTCTTTCTCACCTTTCT CTTCC-3', fwd. 5'-ATTCGCAGTTCATAGGCTATCC-3'.

All primers have been tested by melting curve analysis for specificity for one single amplicon product. qPCR was performed with PerfeCTa SYBR Green FastMix (Quanta Bioscience) according to manufacturer protocol; reactions were carried out using a Chromo4 qPCR detection system (Bio-Rad Laboratories). Results were analyzed using Pfaffl method (Pfaffl 2001).

\section{Statistical analyses of behavioral and biochemical tests}

One-way ANOVA with repeated measures (treatment by days or time) was used for water maze acquisition and open field activity in the young and aged $\mathrm{C} 57 \mathrm{Bl} / 6$ mice experiments. For the AMPK-DN experiment data were analyzed with two-way ANOVA (genotype $\times$ treatment). One-way ANOVA over pool quadrants within groups was applied to analyze the water maze probe trials. Post hoc tests were Fisher's PSLD. Statistical software package used was Statview.

\section{Acknowledgments}

This research was supported by the Intramural Research Program of the NIH, National Institute on Aging (NIA). We thank Chunyan Yuan for assistance with initial experiments and William $\mathrm{H}$. Wood III for assistance with the microarray analysis.

\section{References}

Avraham H, Park SY, Schinkmann K, Avraham S. 2000. RAFTK/ Pyk2-mediated cellular signaling. Cell Signal 12: 123-133.

Baumgartner RN, Rhyne RL, Troup C, Wayne S, Garry PJ. 1992. Appendicular skeletal muscle areas assessed by magnetic resonance imaging in older persons. J Gerontol 47: M67-M72.

Black JE, Isaacs KR, Anderson BJ, Alcantara AA, Greenough WT. 1990. Learning causes synaptogenesis, whereas motor activity causes angiogenesis, in cerebellar cortex of adult rats. Proc Natl Acad Sci 87: $5568-5572$.

Bratic I, Trifunovic A. 2010. Mitochondrial energy metabolism and ageing. Biochim Biophys Acta 1797: 961-967.

Bueno Júnior CR, Pantaleão LC, Voltarelli VA, Bozi LH, Brum PC, Zatz M. 2012. Combined effect of AMPK/PPAR agonists and exercise training in mdx mice functional performance. PLoS One 7: e45699.

Cabeza-Arvelaiz Y, Fleming SM, Richter F, Masliah E, Chesselet MF, Schiestl RH. 2011. Analysis of striatal transcriptome in mice overexpressing human wild-type $\alpha$-synuclein supports synaptic dysfunction and suggests mechanisms of neuroprotection for striatal neurons. Mol Neurodegener 6: 83.

Cheadle C, Vawter MP, Freed WJ, Becker KG. 2003. Analysis of microarray data using $Z$ score transformation. J Mol Diagn 5: 73-81.

Chen Scarabelli C, McCauley RB, Yuan Z, Di Rezze J, Patel D, Putt J, Raddino R, Allebban Z, Abboud J, Scarabelli GM, et al. 2008. Oral administration of amino acidic supplements improves protein and energy profiles in skeletal muscle of aged rats: Elongation of functional performance and acceleration of mitochondrial recovery in adenosine triphosphate after exhaustive exertion. Am J Cardiol 101: $42 \mathrm{E}-48 \mathrm{E}$.

Cheng A, Wan R, Yang JL, Kamimura N, Son TG, Ouyang X, Luo Y, Okun E, Mattson MP. 2012. Involvement of PGC-1 $\alpha$ in the formation and maintenance of neuronal dendritic spines. Nat Comm 3: 1250.

Corsetti G, Pasini E, D'Antona G, Nisoli E, Flati V, Assanelli D, Dioguardi FS, Bianchi R. 2008. Morphometric changes induced by amino acid supplementation in skeletal and cardiac muscles of old mice. Am J Cardiol 101: 26E-34E.

Dagon Y, Avraham Y, Berry EM. 2006. AMPK activation regulates apoptosis, adipogenesis, and lipolysis by eIF $2 \alpha$ in adipocytes. Biochem Biophys Res Commun 340: 43-47.

Dash PK, Orsi SA, Moore AN. 2006. Spatial memory formation and memory-enhancing effect of glucose involves activation of the tuberous sclerosis complex-Mammalian target of rapamycin pathway. J Neurosci 26: 8048-8056.

De S, Zhang Y, Garner JR, Wang SA, Becker KG. 2010. Disease and phenotype gene set analysis of disease-based gene expression in mouse and human. Physiol Genomics 42A: 162-167.

Deng B, Glanzman D, Tidball JG. 2009. Nitric oxide generated by muscle corrects defects in hippocampal neurogenesis and neural differentiation caused by muscular dystrophy. J Physiol 587(Pt 8): $1769-1778$.

Donkor J, Sariahmetoglu M, Dewald J, Brindley DN, Reue K. 2007. Three mammalian lipins act as phosphatidate phosphatases with distinct tissue expression patterns. J Biol Chem 282: 3450-3457.

Edgar R, Domrachev M, Lash AE. 2002. Gene Expression Omnibus: NCBI gene expression and hybridization array data repository. Nucleic Acids Res 30: 207-210.

Erickson CA, Barnes CA. 2003. The neurobiology of memory changes in normal aging. Exp Gerontol 38: 61-69.

Figueiredo PA, Mota MP, Appell HJ, Duarte JA. 2008. The role of mitochondria in aging of skeletal muscle. Biogerontology 9: 67-84.

Fryer LG, Hajduch E, Rencurel F, Salt IP, Hundal HS, Hardie DG, Carling D. 2000. Activation of glucose transport by AMP-activated protein kinase via stimulation of nitric oxide synthase. Diabetes 49: 1978-1985.

Granger R, Wiebe SP, Taketani M, Lynch G. 1996. Distinct memory circuits composing the hippocampal region. Hippocampus 6: 567-578.

Hardie DG. 2011a. Energy sensing by the AMP-activated protein kinase and its effects on muscle metabolism. Proc Nutr Soc 70: 92-99.

Hardie DG. 2011b. Sensing of energy and nutrients by AMP-activated protein kinase. Am J Clin Nutr 93: 891S-896S.

Hardie DG, Ross FA, Hawley SA. 2012. AMPK: A nutrient and energy sensor that maintains energy homeostasis. Nat Rev Mol Cell Biol 13: $251-262$

Harris RA, Bowker-Kinley MM, Wu P, Jeng J, Popov KM. 1997. Dihydrolipoamide dehydrogenase-binding protein of the human pyruvate dehydrogenase complex. DNA-derived amino acid sequence, expression, and reconstitution of the pyruvate dehydrogenase complex. J Biol Chem 272: 19746-19751.

Hillman CH, Erickson KI, Kramer AF. 2008. Be smart, exercise your heart: Exercise effects on brain and cognition. Nat Rev Neurosci 9: 58-65.

Kobilo T, van Praag H. 2012. Muscle fatigue and cognition: What is the link? Front Physiol 3: 14. 
Kobilo T, Yuan C, van Praag H. 2011. Endurance factors improve hippocampal neurogenesis and spatial memory in mice. Learn Mem 18: $103-107$.

Lang T, Streeper T, Cawthon P, Baldwin K, Taaffe DR, Harris TB. 2010 Sarcopenia: Etiology, clinical consequences, intervention, and assessment. Osteoporos Int 21: 543-559.

Ljubicic V, Hood DA. 2009. Diminished contraction-induced intracellular signaling towards mitochondrial biogenesis in aged skeletal muscle. Aging Cell 8: 394-404.

Mansouri A, Muller FL, Liu Y, Ng R, Faulkner J, Hamilton M, Richardson A, Huang TT, Epstein CJ, Van Remmen H. 2006. Alterations in mitochondrial function, hydrogen peroxide release and oxidative damage in mouse hind-limb skeletal muscle during aging. Mech Ageing Dev 127: 298-306.

Marangos PJ, Loftus T, Wiesner J, Lowe T, Rossi E, Browne CE, Gruber HE. 1990. Adenosinergic modulation of homocysteine-induced seizures in mice. Epilepsia 31: 239-246.

März P, Probst A, Lang S, Schwager M, Rose-John S, Otten U, Ozbek S. 2004. Ataxin-10, the spinocerebellar ataxia type 10 neurodegenerative disorder protein, is essential for survival of cerebellar neurons. J Biol Chem 279: 35542-35550.

Mattson MP. 2012. Energy intake and exercise as determinants of brain health and vulnerability to injury and disease. Cell Metab 16: 706-722.

Mattson MP, Magnus T. 2006. Ageing and neuronal vulnerability. Nat Rev Neurosci 7: 278-294.

McDermott C, Cardoso MC, Yu YT, Andres V, Leifer D, Krainc D, Lipton SA, Nadal-Ginard B. 1993. hMEF2C gene encodes skeletal muscle- and brain-specific transcription factors. J Mol Cell Biol 13: 2564-2577.

Mironova YA, Giger RJ. 2013. Where no synapses go: Gatekeepers of circuit remodeling and synaptic strength. Trends Neurosci 36: 363-373.

Miura S, Kai Y, Kamei Y, Bruce CR, Kubota N, Febbraio MA, Kadowaki T, Ezaki O. 2009. $\alpha 2$-AMPK activity is not essential for an increase in fatty acid oxidation during low-intensity exercise. Am J Physiol Endocrinol Metab 296: E47-E55.

Morris RG, Garrud P, Rawlins JN, O'Keefe J. 1982. Place navigation impaired in rats with hippocampal lesions. Nature 24: 681-683.

Nagata A, Suzuki Y, Igarashi M, Eguchi N, Toh H, Urade Y, Hayaishi O. 1991. Human brain prostaglandin D synthase has been evolutionarily differentiated from lipophilic-ligand carrier proteins. Proc Natl Acad Sci 88: $4020-4024$.

Nakamura T, Komiya M, Sone K, Hirose E, Gotoh N, Morii H, Ohta Y, Mori N. 2002. Grit, a GTPase-activating protein for the Rho family, regulates neurite extension through association with the TrkA receptor and N-Shc and CrkL/Crk adapter molecules. Mol Cell Biol 22: $8721-8734$.

Nakamura K, Nakano S, Miyoshi T, Yamanouchi K, Matsuwaki T, Nishihara M. 2012. Age-related resistance of skeletal muscle-derived progenitor cells to SPARC may explain a shift from myogenesis to adipogenesis. Aging 4: 40-48.

Narkar VA, Downes M, Yu RT, Embler E, Wang YX, Banayo E, Mihaylova MM, Nelson MC, Zou Y, Juguilon H, et al. 2008. AMPK and

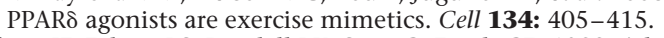

Nelson JF, Felicio LS, Randall PK, Sims C, Finch CE. 1982. A longitudinal study of estrous cyclicity in aging C57BL/6J mice: I. Cycle frequency, length and vaginal cytology. Biol Reprod 27: 327-339.

Ornatsky OI, McDermott JC. 1996. MEF2 protein expression, DNA binding specificity and complex formation, and transcriptional activity in muscle and non-muscle cells. J Biol Chem 271: 24927-24933.

Pedersen PL, Amzel LM. 1993. ATP synthases. Structure, reaction center, mechanism, and regulation of one of nature's most unique machines. J Biol Chem 268: 9937-9940.

Pedersen BK, Febbraio MA. 2012. Muscles, exercise and obesity: Skeletal muscle as a secretory organ. Nat Rev Endocrinol 8: 4574-4565.

Pedersen BK, Steensberg A, Fischer C, Keller C, Keller P, Plomgaard P, Febbraio M, Saltin B. 2003. Searching for the exercise factor: Is IL-6 a candidate? J Muscle Res Cell Motil 24: 113-119.
Peterson CM, Johannsen DL, Ravussin E. 2012. Skeletal muscle mitochondria and aging: A review. J Aging Res 2012: 194821.

Pfaffl MW. 2001. A new mathematical model for relative quantification in real-time RT-PCR. Nucleic Acids Res 29: e45.

Potter WB, O'Riordan KJ, Barnett D, Osting SM, Wagoner M, Burger C, Roopra A. 2010. Metabolic regulation of neuronal plasticity by the energy sensor AMPK. PLoS One 5: 8996.

Qiang W, Weiqiang K, Qing Z, Pengju Z, Yi L. 2007. Aging impairs insulin-stimulated glucose uptake in rat skeletal muscle via suppressing AMPK $\alpha$. Exp Mol Med 39: 535-543.

Reznick RM, Zong H, Li J, Morino K, Moore IK, Yu HJ, Liu ZX, Dong J, Mustard KJ, Hawley SA, et al. 2007. Aging-associated reductions in AMP-activated protein kinase activity and mitochondrial biogenesis. Cell Metab 5: 151-156.

Sakuma K, Yamaguchi A. 2011. The recent understanding of the neurotrophin's role in skeletal muscle adaptation. J Biomed Biotechnol 2011: 201696.

Segatto O, Anastasi S, Alemà S. 2011. Regulation of epidermal growth factor receptor signalling by inducible feedback inhibitors. J Cell Sci 124(Pt 11): $1785-1793$.

Sun X-y, Hayashi Y, Xu S, Kanou Y, Takagishi Y, Tang Y-p, Murata Y. 2011 Inactivation of the Rcan 2 gene in mice ameliorates the age- and diet-induced obesity by causing a reduction in food intake. PLoS One 6: e14605.

Teng FY, Tang BL. 2008. Cell autonomous function of Nogo and reticulons: The emerging story at the endoplasmic reticulum. J Cell Physiol 216: 303-308.

Teng FY, Tang BL. 2013. Nogo/RTN4 isoforms and RTN3 expression protect SH-SY5Y cells against multiple death insults. Mol Cell Biochem 384: $7-19$.

Terry AV Jr. 2009. Spatial navigation (water maze) tasks. In Methods of behavior analysis in neuroscience, 2nd ed. (ed. Buccafusco JJ), Chapter 13. CRC Press, Boca Raton, FL.

Thomson DM, Winder WW. 2009. AMP-activated protein kinase control of fat metabolism in skeletal muscle. Acta Physiol (Oxf) 196: $147-154$.

van Praag H. 2008. Neurogenesis and exercise: Past and future directions. Neuromolecular Med 10: 128-140.

van Rooij E, Quiat D, Johnson BA, Sutherland LB, Qi X, Richardson JA, Kelm RJ Jr., Olson EN. 2009. A family of microRNAs encoded by myosin genes governs myosin expression and muscle performance. Dev Cell 17: $662-673$.

Walker JE. 1992. The NADH: Ubiquinone oxidoreductase (complex I) of respiratory chains. Q Rev Biophys 25: 253-324.

Wu QF, Yang L, Li S, Wang Q, Yuan XB, Gao X, Bao L, Zhang X. 2012. Fibroblast growth factor 13 is a microtubule-stabilizing protein regulating neuronal polarization and migration. Cell 149: 1549-1564.

Xu Y, Zhang HT, O’Donnell JM. 2011. Phosphodiesterases in the central nervous system: Implications in mood and cognitive disorders. Handb Exp Pharmacol 204: 447-485.

Zhang Y, De S, Garner JR, Smith K, Wang SA, Becker KG. 2010. Systematic analysis, comparison, and integration of disease based human genetic association data and mouse genetic phenotypic information. BMC Med Genomics 3: 1 .

Zong H, Ren JM, Young LH, Pypaert M, Mu J, Birnbaum MJ, Shulman GI. 2002. AMP kinase is required for mitochondrial biogenesis in skeletal muscle in response to chronic energy deprivation. Proc Natl Acad Sci 99: $15983-15987$.

Zwetsloot KA, Westerkamp LM, Holmes BF, Gavin TP. 2008. AMPK regulates basal skeletal muscle capillarization and VEGF expression, but is not necessary for the angiogenic response to exercise. J Physiol 586: $6021-6035$.

Received September 22, 2013; accepted in revised form November 16, 2013. 


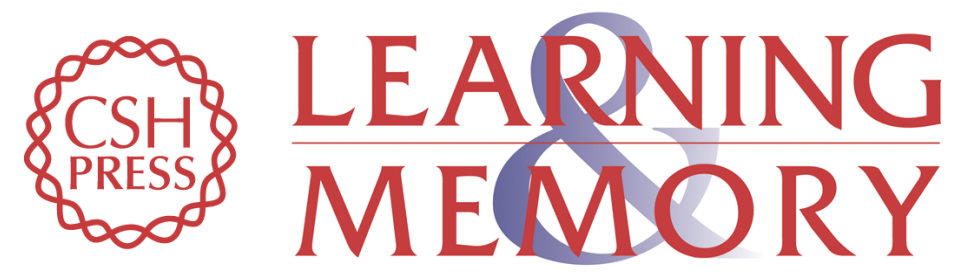

\section{AMPK agonist AICAR improves cognition and motor coordination in young and aged mice}

Tali Kobilo, Davide Guerrieri, Yongqing Zhang, et al.

Learn. Mem. 2014, 21:

Access the most recent version at doi:10.1101/Im.033332.113

References This article cites 66 articles, 13 of which can be accessed free at: http://learnmem.cshlp.org/content/21/2/119.full.html\#ref-list-1

Creative This article is distributed exclusively by Cold Spring Harbor Laboratory Press for the Commons License first 12 months after the full-issue publication date (see

http://learnmem.cshlp.org/site/misc/terms.xhtml). After 12 months, it is available under a Creative Commons License (Attribution-NonCommercial 3.0 Unported), as described at http://creativecommons.org/licenses/by-nc/3.0/.

Email Alerting Receive free email alerts when new articles cite this article - sign up in the box at the Service top right corner of the article or click here. 